\title{
Developing a Model to Simulate the Effect of Hypothermia on Cerebral Blood Flow and Metabolism
}

\author{
Joshua Russell-Buckland and Ilias Tachtsidis
}

\begin{abstract}
Hypoxic ischemic encephalopathy (HIE) is a significant cause of death and neurological disability in newborns. Therapeutic hypothermia at $33.5^{\circ} \mathrm{C}$ is one of the most common treatments in HIE and generally improves outcome; however $45-55 \%$ of injuries still result in death or severe neurodevelopmental disability. We have developed a systems biology model of cerebral oxygen transport and metabolism to model the impact of hypothermia on the piglet brain (the neonatal preclinical animal model) tissue physiology. This computational model is an extension of the BrainSignals model of the adult brain. The model predicts that during hypothermia there is a $5.1 \%$ decrease in cerebral metabolism, $1.1 \%$ decrease in blood flow and $2.3 \%$ increase in cerebral tissue oxygenation saturation. The model can be used to simulate effects of hypothermia on the brain and to help interpret bedside recordings.
\end{abstract}

Keywords Broadband NIRS $\cdot$ Hypothermia $\cdot$ Systems biology

\section{Introduction}

Hypoxic-ischaemic encephalopathy (HIE), often resulting from intrapartum hypoxic-ischemic (HI) injury, is a significant cause of death and morbidity. Somewhere between 700,000 deaths amongst neonates can be attributed to HIE alone [1]. Following neonatal HI injury or birth asphyxia, infants are treated with therapeutic hypothermia at a temperature of $33.5^{\circ} \mathrm{C}$, as well as a variety of medications such as morphine and atracurium [2]. However, 45-55\% of cases treated with hypothermia end with death or moderate to severe neurodevelopmental disability $[1,3]$. The brain of neonates undergoing hypothermia treatment is monitored through a variety of methods such as electro-encephalography (EEG) [4] and

J. Russell-Buckland $(\bowtie) \cdot$ I. Tachtsidis

Biomedical Optics Research Laboratory, University College London, London, UK

e-mail: joshua.russell-buckland.15@ucl.ac.uk 
near-infrared spectroscopy (NIRS) [2, 5]. Additionally, in the few hospitals with appropriate facilities, when the infant is stable enough (usually a week after birth) and following treatment they image the brain with magnetic resonance spectroscopy (MRS) [6]. The collected multimodal data can provide not only diagnostic and prognostic information but also insights on the mechanisms of the injury.

Our approach to analyse this multimodal data has been multifaceted and includes the development and application of (1) a physiology-informed 'mathematical model' of the cerebral circulation under a systems biology approach, which is specially designed for the interpretation of NIRS signals [7-12]; and (2) 'signal processing' methods quantifying the interrelationships between signals both in time- and frequency-domain to quantify indices of cerebral blood flow, oxygenation and metabolic regulation $[2,5]$.

The development of systems biology-based models begin with the 'BRAINCIRC' model in 2005 [7]. This built on an earlier circulatory model by Ursino and Lodi [13] and combined models for the biophysics of the circulatory system, the brain metabolic biochemistry and the function of vascular smooth muscle. This model was succeeded by the 'BrainSignals' model [8], which simplified the previous BRAINCIRC model and added a submodel of mitochondrial metabolism. Additional versions were then developed from this, such as the 'BrainPiglet' model [9] which was developed to interpret data collected from a piglet model. It involved modifying the default values for 11 of the 107 parameters used and was extended to include simulated measurements for magnetic resonance spectroscopy values, which are available for the piglet model. In 2015, Caldwell et al. modified the BrainSignals model to produce the 'BrainSignals Revisited' model [10]. This made various simplifications to the BrainSignals model to reduce complexity and decrease the time taken to run a simulation, whilst being able to reproduce the same results and behaviour of the original model. This reduced model was later extended in 2016 to simulate scalp haemodynamics to produce the 'BSX' model [11]. This could be used to investigate the potential for systemic haemodynamics to confound fNIRS haemoglobin signals. The BrainPiglet model was also extended to model cell death following HIE producing the 'BrainPigletHI' model [12]. This history is shown schematically in Fig. 1.

However, the existing mathematical models have been developed to model brain physiology under 'typical' conditions, particularly at normal body temperature. In order to properly interpret data collected during therapeutic hypothermia, the models need to be able to reflect these biological conditions. It has been observed that both cerebral metabolic rate of oxygen $\left(\mathrm{CMRO}_{2}\right)$ and cerebral blood flow (CBF) in piglets decrease with reduced body temperature [14]. For reliable inferences to be made through the use of systems biology models, they must be able to simulate this behaviour.

We present a new systems biology model of the piglet brain, a common animal model for the human neonatal brain, which incorporates temperature effects in the brain tissue physiology. This is an expansion of the BrainPigletHI model, as seen in [12]. The model is used to demonstrate how brain tissue temperature impacts brain physiology steady state behaviour for changing blood pressure, arterial oxygen saturation and partial pressure of $\mathrm{CO}_{2}$. It is then shown how decreasing temperature impacts a variety of measurable signals. 


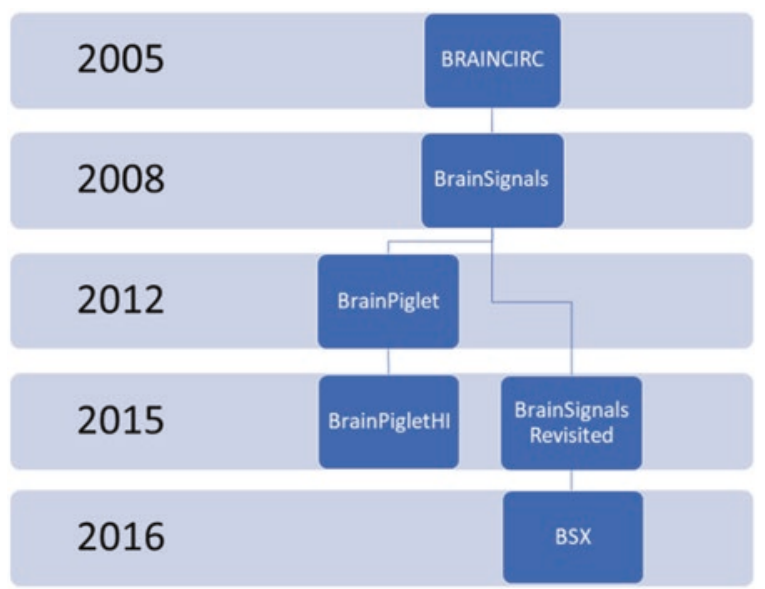

Fig. 1 History of the brainsignals family of models. A general overview of the brainsignals model history. The first model was the BRAINCIRC model in 2005, followed by the brainsignals model in 2008. This model was adapted for the physiology of piglets, a common neonatal preclinical animal model, in 2012 producing the brainpiglet model. The brainpiglet model was modified to include cell death in 2015, to produce the brainpigletHI model. Also in 2015, the brainsignal model was refactored for speed and clarity, producing the brainsignals revisited model. This was extended in 2016 to include scalp haemodynamics, producing the BSX model

\section{Methods}

Temperature is already incorporated into the BrainPiglet model as a constant value within the proportionality constant $Z=R T / F$, where $R$ is the ideal gas constant, $F$ is the Faraday constant and $T$ is absolute body temperature. The constant $Z$ is used within the model to determine the equilibrium constant for a variety of metabolic reactions, such as electron transfer within mitochondria. By making $Z$ a function of temperature, temperature modulated electron transfer is incorporated into the metabolic submodel.

Temperature also impacts the rate of other reactions and processes, such as diffusion or Michaelis-Menten type behaviour. Orlowski et al. [15] expanded an existing model of cellular metabolism by incorporating temperature dependence into the rates of reaction and ion diffusion and a similar approach is taken here. Reaction rates, Michaelis-Menten rate constants and diffusion rates are modified by a quantity

$$
k_{i, \text { new }}=k_{i, \text { previous }} \times Q_{10}^{\frac{T_{\text {new }}-T_{\text {previous }}}{10 .}}
$$

where $k_{i, n e w}$ is the new rate constant for reaction $i$ at the new temperature $T_{\text {new }}$, $k_{i, \text { previous }}$ is the rate constant at temperature $T_{\text {previous }}$, and $Q_{10}$ is the temperature 


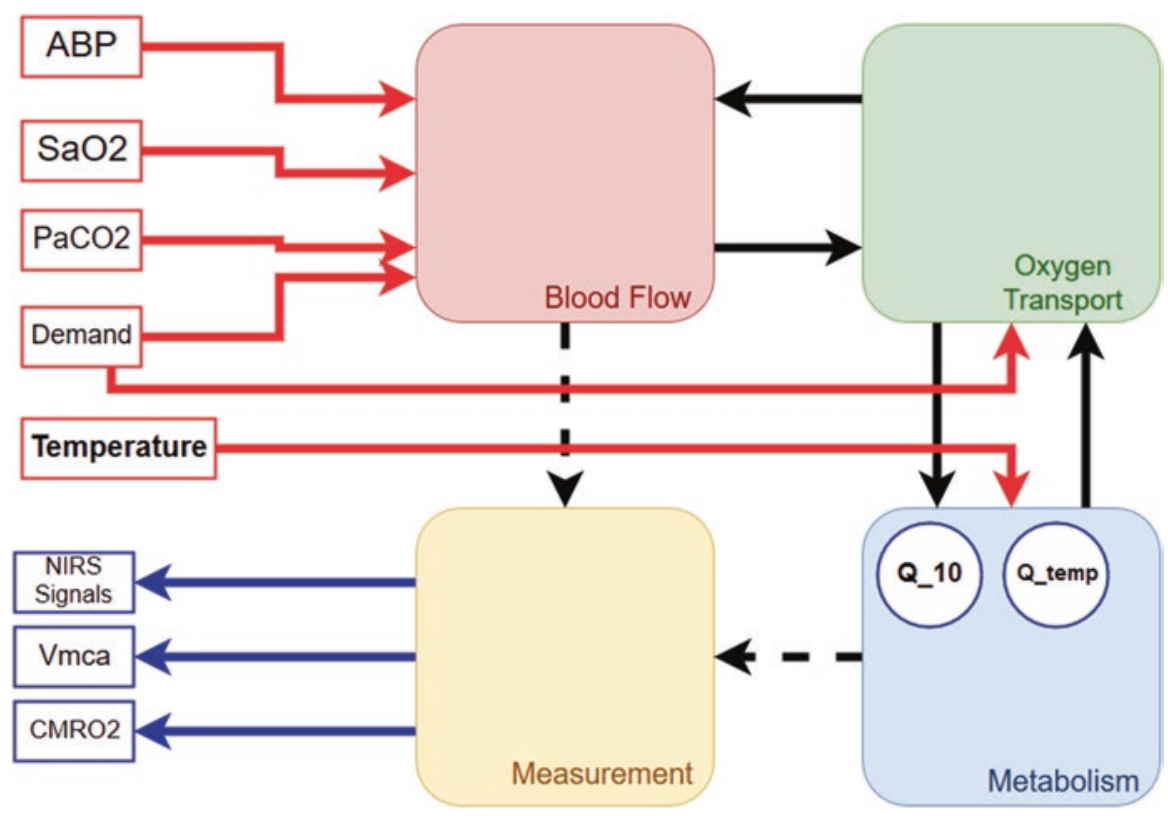

Fig. 2 General structure of the brainpiglet hypothermia model. Model inputs, arterial blood pressure (ABP, arterial oxygen saturation $(\mathrm{SaO} 2)$, partial pressure of $\mathrm{CO}_{2}(\mathrm{PaCO} 2)$ and demand, and outputs are shown, as well as each of the four sub-models and the general relations between each. New additions are shown in bold. Temperature is added as an input to the model. Q_10, a measure of the change in reaction rate when increasing temperature by $10^{\circ} \mathrm{C}$, is a new parameter. Q_temp, the factor by which a reaction rate is modified when changing temperature, is added as a new internal state variable local to the metabolism submodel

coefficient, defined as the ratio of reaction rates measured for the same reaction at two temperatures $10{ }^{\circ} \mathrm{C}$ apart, with an initial default value of 2.23, as per [15], but an expected final value within the range of approximately 2-3 as per most biological reactions [16]. Typically, $T_{\text {previous }}$ will be assumed to be normal body temperature of $37^{\circ} \mathrm{C}$, but this can be set as a model parameter. For simplification purposes, within the model the temporary variable $Q_{\text {temp }}=Q_{10}^{\frac{T_{\text {new }}-T_{\text {previous }}}{10}}$ is defined. Figure 2 shows a simplified schematic of the model structure, with the new model additions of temperature, $Q_{10}$ and $Q_{\text {temp }}$ shown in bold.

Models were then tested and verified through steady state simulations, at $37^{\circ} \mathrm{C}$, $35^{\circ} \mathrm{C}$ and $33.5^{\circ} \mathrm{C}$, varying arterial blood pressure, arterial oxygen saturation and partial pressure of $\mathrm{CO}_{2}$. Following this, all model inputs except temperature were kept at their default values, whilst temperature was decreased between $37{ }^{\circ} \mathrm{C}$ and $33.5^{\circ} \mathrm{C}$ for varying values of $Q_{10}$. 


\section{Results}

Steady state simulations were carried out at three temperatures $\left(33.5^{\circ} \mathrm{C}, 35^{\circ} \mathrm{C}\right.$ and $37^{\circ} \mathrm{C}$ ) to investigate the effects of hypothermia on brain vascular autoregulation. The three main measured inputs of arterial blood pressure $\left(\mathrm{P}_{\mathrm{a}}\right)$, arterial oxygen saturation $\left(\mathrm{SaO}_{2}\right)$ and partial pressure of $\mathrm{CO}_{2}\left(\mathrm{PaCO}_{2}\right)$ were both increased and decreased with $\mathrm{CBF}$ simulated at the end of $100 \mathrm{~s}$ at each step change. Figure 3 shows that cerebral autoregulation is preserved at all temperatures and for both increasing and decreasing changes in $\mathrm{P}_{\mathrm{a}}, \mathrm{SaO}_{2}$ and $\mathrm{PaCO}_{2}$.

Following this, the effect of decreasing temperature on physiology within the model was investigated through further use of steady state simulations. Temperature was decreased to $33.5^{\circ} \mathrm{C}$ with all other inputs kept at baseline values for a range of $\mathrm{Q}_{10}$ values. Without a known value of $\mathrm{Q}_{10}$ it was important to investigate a range of values to ensure that the full range of model behaviour could be considered. As with the autoregulation simulations, output values were simulated after $100 \mathrm{~s}$ at each temperature step. Outputs considered are: (a) brain tissue oxygenation saturation $\left(\mathrm{StO}_{2}\right)$, (b) $\mathrm{CBF}$, (c) brain tissue haemoglobin differences changes ( $\triangle \mathrm{HbD}=$ oxyhaemoglobin-deoxyhaemoglobin), (d) brain tissue changes in the concentration of the oxidation of cytochrome- $c$-oxidase $(\triangle \mathrm{oxCCO})$, and (e) cerebral metabolic rate of oxygen $\left(\mathrm{CMRO}_{2}\right)$. Figure 4 shows that, across all outputs except $\mathrm{StO}_{2}$ and $\Delta \mathrm{HbD}$, the output decreases linearly, whilst for $\mathrm{StO}_{2}$ and $\Delta \mathrm{HbD}$ an increase in value is, instead, observed as temperature decreases. The rate of change increases with $\mathrm{Q}_{10}$.
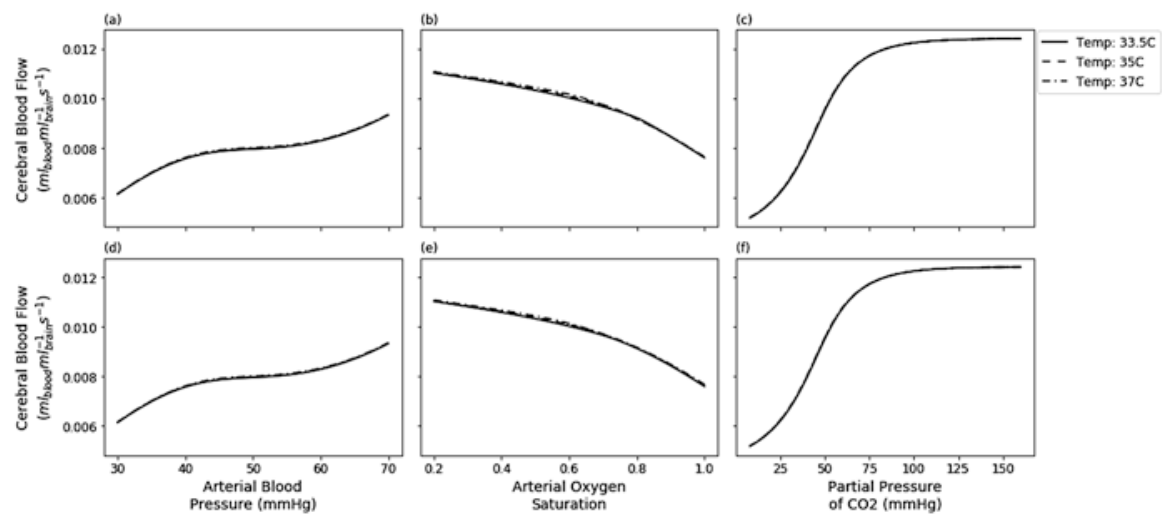

Fig. 3 Steady states curves showing cerebral blood flow against increasing (a) arterial blood pressure; (b) arterial oxygen saturation; (c) partial pressure of $\mathrm{CO}_{2}$ and decreasing (d) arterial blood pressure; (e) arterial oxygen saturation; (f) partial pressure of $\mathrm{CO}_{2}$ for temperatures of $33.5{ }^{\circ} \mathrm{C}, 35{ }^{\circ} \mathrm{C}$ and $37^{\circ} \mathrm{C}$. It can be seen in all figures that autoregulation is preserved across all three temperatures and there is little difference between increasing and decreasing values of input 

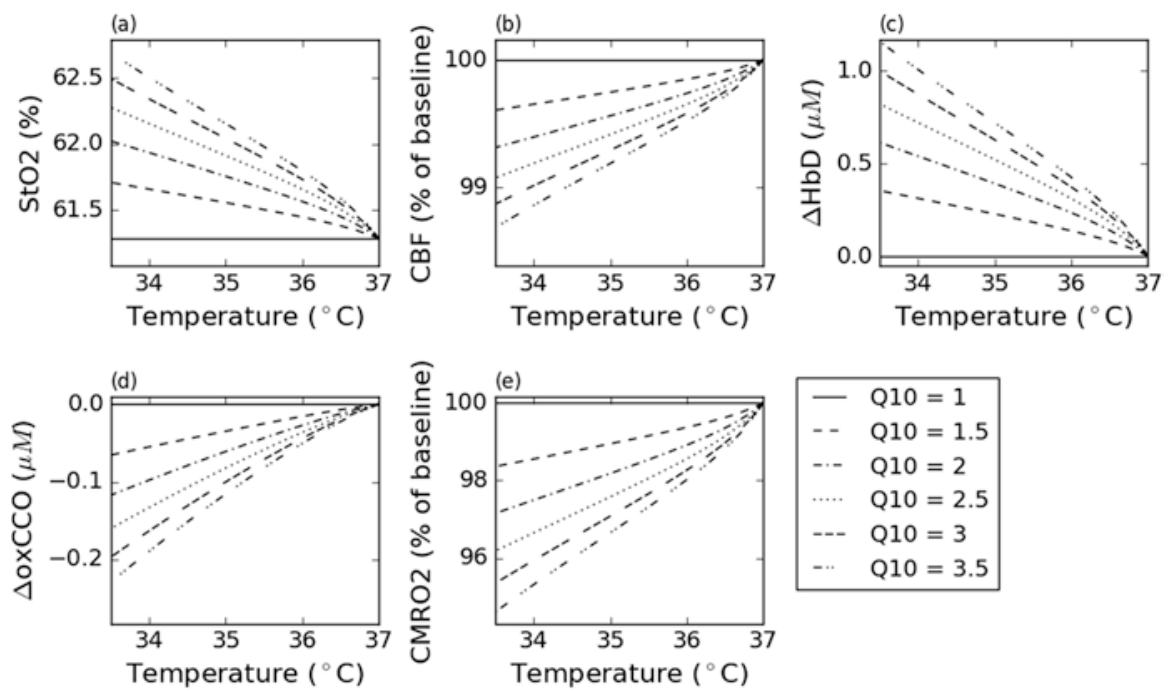

Fig. 4 Steady state curves showing the effect of decreasing temperature on (a) total oxygenation index; (b) cerebral blood flow; (c)velocity of the middle cerebral artery; (d) change in concentration of cytochrome-c-oxidase; (e) cerebral metabolic rate of oxygen for varying values of $\mathbf{Q}_{10}$. Steady state simulations show expected behaviour across outputs, where information is available to confirm, such as the decrease cerebral blood flow and cerebral metabolic rate of oxygen with decreasing temperature down to $33.5^{\circ} \mathrm{C}$. The observed change is linear, with the rate of change increasing with the $\mathrm{Q}_{10}$ value

Considering $\mathrm{Q}_{10}$ values of 2.5 , which is in the middle of the range given previously for most biological reactions, and 3.5 , which is the largest value considered, CBF decreases by $1.1 \%$ and $1.6 \%$, respectively whilst the $\mathrm{CMRO}_{2}$ decrease by $5.1 \%$ and $7.1 \%$ respectively. $\mathrm{StO}_{2}$ increases by $2.3 \%$ and $3.2 \%$ of normothermic values respectively.

\section{Discussion}

We have expanded an existing systems biology model of the piglet brain physiology to include the impact of hypothermia. This has been done by introducing a minimal number of new parameters and inputs and is able to produce qualitatively reasonable results. Simulations of cerebral autoregulation agree with the findings of Lee et al. who found that hypothermia did not change autoregulation within a piglet model for both hyper- and hypotensive changes [17] (Fig. 3).

When considering the effect of temperature on various measurable outcomes, we see that both $\mathrm{CBF}$ and $\mathrm{CMRO}_{2}$ decrease with decreasing temperature, as found by Ehrlich et al. [9]. Whilst the model changes are smaller than those found by Ehrlich et al., the final cooled temperature is not as low and it is not the intention of the 
model to simulate below a temperature of $33.5^{\circ} \mathrm{C}$. We expect changes in brain physiology at this temperature to be subtle but important. Below this temperature damage to the brain may occur causing a change in brain physiology not captured by this model.

Systems biology models were previously used by our group to investigate the effect of HI in the piglet model [12]; however, they were not able to incorporate the impact of therapeutic hypothermia alongside this injury. In particular, it was found that the pre- and post-injury brain is sufficiently different to require refitting of the model and a number of measurable differences could be explained through the incorporation of a parameter representing cell death within the brain. By combining this previously developed approach with the new hypothermia model, it may be possible to further explain other behaviour. We aim to apply the model to broadband NIRS data, including cytochrome-c-oxidase measurements, collected within the group that show differing responses to hypothermia in piglets who received the same injury. We will apply the model to data collected from human neonates, where we expect the same model structure to apply but with different model parameters to reflect the difference in human and pig physiology.

Acknowledgments This research was funded by The Wellcome Trust, grant 104580/Z/14/Z

\section{References}

1. Hassell KJ, Ezzati M, Alonso-Alconada D et al (2015) New horizons for newborn brain protection: enhancing endogenous neuroprotection. Arch Di Child Fetal Neonatal Ed 100:F541F552

2. Bale G, Mitra S, de Roever I et al (2018) Oxygen dependency of mitochondrial metabolism indicates outcome of newborn brain injury. J Cerebral Blood Flow Metab 0(00):1-13

3. Gluckman P, Wyatt J, Azzopardinna D et al (2005) Selective head cooling with mild systemic hypothermia after neonatal encephalopathy: multicentre randomized trial. Lancet 365(9460):663-670

4. Murray D, Boylan GB, Ryan AC et al (2009) Early EEG findings in hypoxic-ischemic encephalopathy predict outcomes at 2 years. Pediatrics 124:e459-e467. https://doi.org/10.1542/ peds.2008-2190

5. Bale G, Mitra S, Meek J et al (2014) A new broadband near-infrared spectroscopy system for in-vivo measurements of cerebral cytochrome-c-oxidase changes in neonatal brain injury. Biomed Opt Express 5:3450-3466. https://doi.org/10.1364/BOE.5.003450

6. Vries L, Groenendaal F (2010) Patterns of neonatal hypoxic-ischaemic brain injury. Neuroradiology 52:555-566. https://doi.org/10.1007/s00234-010-0674-9

7. Banaji M, Tachtsidis I, Delpy D, Baigent S (2005) A physiological model of cerebral blood flow control. Math Biosci 194(2):125-173. https://doi.org/10.1016/j.mbs.2004.10.005

8. Banaji M, Mallet A, Elwell CE, Nicholls P, Cooper CE (2008) A model of brain circulation and metabolism: NIRS signal changes during physiological challenges. PLoS Comput Biol 4(11):e1000212

9. Moroz T, Banaji M, Robertson NJ, Cooper CE, Tachtsidis I (2012) Computational modelling of the piglet brain to simulate near-infrared spectroscopy and magnetic resonance spectroscopy data collected during oxygen deprivation. J R Soc Interface 9(72):1499-1509

10. Caldwell M, Hapuarachchi T, Highton D et al (2015) BrainSignals revisited: simplifying a computational model of cerebral physiology. PLoS One 10:e0126695. https://doi.org/10.1371/ journal.pone.0126695 
11. Caldwell M, Scholkmann F, Wolf U, Wolf M, Elwell C, Tachtsidis I (2016) Modelling confounding effects from extracerebral contamination and systemic factors on functional near-infrared spectroscopy. NeuroImage 143:91-105. https://doi.org/10.1016/j.neuroimage.2016.08.058

12. Caldwell M, Moroz T, Hapuarachchi T et al (2015) Modelling blood flow and metabolism in the preclinical neonatal brain during and following hypoxic-Ischaemia. PLoS One 10:e0140171. https://doi.org/10.1371/journal.pone.0140171

13. Ursino M, Lodi CA (1997) A simple mathematical model of the interaction between intracranial pressure and cerebral hemodynamics. J Applied Physiology 82(4):1256-1269

14. Ehrlich MP, McCullough JN, Zhang N et al Effect of hypothermia on cerebral blood flow and metabolism in the pig. Ann Thoracic Surg 73:191-197

15. Orlowski P, McConnell FK, Payne S (2014 Feb) A mathematical model of cellular metabolism during ischemic stroke and hypothermia. IEEE Trans Biomed Eng 61(2):484-490

16. Reyes B, Pendergast J, Yamazaki S (2008) Mammalian peripheral circadian oscillators are temperature compensated. J Biol Rhythm 23:95-98. https://doi.org/10.1177/0748730407311855

17. Lee JK, Brady KM, Mytar JO et al (2011) Cerebral blood flow and cerebrovascular autoregulation in a swine model of pediatric cardiac arrest and hypothermia. Crit Care Med 39(10):2337-2345. https://doi.org/10.1097/ccm.0b013e318223b910

Open Access This chapter is licensed under the terms of the Creative Commons Attribution 4.0 International License (http://creativecommons.org/licenses/by/4.0/), which permits use, sharing, adaptation, distribution and reproduction in any medium or format, as long as you give appropriate credit to the original author(s) and the source, provide a link to the Creative Commons license and indicate if changes were made.

The images or other third party material in this chapter are included in the chapter's Creative Commons license, unless indicated otherwise in a credit line to the material. If material is not included in the chapter's Creative Commons license and your intended use is not permitted by statutory regulation or exceeds the permitted use, you will need to obtain permission directly from the copyright holder. 